\title{
Side emissions during EC injection for PDI studies in FTU tokamak
}

Alessandro Bruschi ${ }^{1, *}$, Edoardo Alessi ${ }^{1}$, Benedetta Baiocchi ${ }^{1}$, William Bin $^{1}$, Ocleto D'Arcangelo ${ }^{2}$, Francesco Fanale ${ }^{1}$, Lorenzo Figini ${ }^{1}$, Cristian Galperti ${ }^{3}$, Saul Garavaglia ${ }^{1}$, Giuseppe Gittini ${ }^{1}$, Gustavo Granucci ${ }^{1}$, Giovanni Grosso ${ }^{1}$, Lev Lubyako $^{4}$, Cristina Mazzotta ${ }^{2}$, Vittoria Mellera ${ }^{1}$, Alessandro Moro ${ }^{1}$, Francesco Orsitto ${ }^{5}$, Fabrizio Pallotta ${ }^{1}$, Giuliano Rocchi $^{2}$, Alessandro Simonetto ${ }^{1}$, Umberto Tartari $^{1}$, Onofrio Tudisco ${ }^{2}$

${ }^{1}$ Istituto di Fisica del Plasma, Consiglio Nazionale delle Ricerche, via R. Cozzi 53, 20125 Milano, Italy

${ }^{2}$ ENEA Fusion and Nuclear Safety Department, C.R.Frascati, via E. Fermi 45, 00044 Frascati (Roma), Italy

${ }^{3}$ Swiss Plasma Center, Ecole Polytéchnique Fédérale de Lausanne, Lausanne, Switzerland

${ }^{4}$ Institute of Applied Physics, 46 Ulyanov st., Nizhny Novgorod, 603950, Russia

${ }^{5}$ Consorzio CREATE, Università degli Studi di Napoli, Napoli, Italy

\begin{abstract}
The evidence of Parametric Decay Instabilities (PDI) excited by the ECH power injected in OMode has been explored in FTU Tokamak, using the Collective Thomson Scattering (CTS) diagnostic. The experiments show evidences to support the hypothesis of low-threshold excitation of waves generated by PDI mechanisms, formerly proposed in the case of 2nd harmonic X-mode injection in TEXTOR and ASDEX-U. Theoretical analysis predicts low-threshold parametric decay also for O-mode pump-wave injection, which can be injected in FTU at frequencies close to the first Harmonic EC resonance. Experiments were made at different magnetic fields, injecting the $140 \mathrm{GHz}$ probe and observing the emission from the second antenna of the EC launcher in poloidally symmetric and asymmetric configurations, in presence of MHD islands. The signal is detected by the CTS radiometers, with a fast digitizer allowing the spectral reconstruction at very fine time and frequency scales. Different types of emissions are studied in detail, comparing them with the magnetic island rotation frequency in different plasma conditions. In order to locate the plasma volume originating the emissions, a new antenna and receiving line has been installed.
\end{abstract}

\section{Introduction}

The presence of emissions due to parametric decay instabilities (PDI) during the injection of the ECH power at the $1{ }^{\text {st }}$ Harmonic O-Mode has been investigated in the FTU Tokamak, using the renewed Collective Thomson Scattering (CTS) diagnostic [1-3].

The aim was to add experimental evidences to confirm the hypotheses of low-threshold excitation of waves generated by PDI mechanisms based on the observation of back-scattered power in the case of $2^{\text {nd }}$ harmonic Xmode injection in TEXTOR [4 and reference therein], and on other observations in ASDEX-U [5]. Theoretical analysis [6,7] predicts the possibility of low-threshold parametric decay also for O-mode pump-wave injection, which can be explored at frequencies close to the first Harmonic resonance in FTU.

Experiments were made at magnetic fields of $4.7 \mathrm{~T}$ and 3.6 $\mathrm{T}$, injecting the $140 \mathrm{GHz}$ probe and observing the emission from the second antenna of the EC launcher in symmetric and asymmetric configurations (see section 1.1), in presence of MHD islands mainly stimulated by $\mathrm{Ne}$ injection. The signal is split in two orthogonal polarizations and detected with two channels of the CTS radiometer, with a fast digitizer connected to the IF of the two front-ends, recording the time traces of the signal at rates 3 to $6 * 10^{9}$ samples/s. The spectral reconstruction can be done offline by finely tuning the time windows of the Fourier Transform of samples, obtaining the possibility of very high time and frequency resolution. Different types of emissions have been reported [8], some of which are studied in further detail, to exclude gyrotron spurious emission and to verify the synchronism with the magnetic island rotation. The plasma conditions in which the various types of emissions are found have been extensively studied. In order to locate the plasma volume originating the emissions, which would help to identify the causing phenomena, a new receiving antenna with a transmission line independent from the ECH lines and with a higher decoupling with respect to the probe has been installed.

\subsection{Plasma and experimental conditions}

The work has been performed in two main plasma conditions, the first at 4.7 $\mathrm{T}$ magnetic field in the vessel centre, with the resonant layer for the probe frequency shifted a few cm toward the plasma high-field side (see 
Fig.1, left), and the second at $3.6 \mathrm{~T}$ in the vessel centre, with the first-harmonic resonant layer for the probe frequency shifted at the plasma edge towards the highfield side (Fig.1, right). The plasma total current was respectively $500 \mathrm{kA}$ and $350 \mathrm{kA}$ in the two cases, in order to keep a similar q-profile, with similar location of the $\mathrm{m}: \mathrm{n}=2: 1$ tearing mode. The MHD island at $\mathrm{q}=2$ is often already present in the plasma, but for our purposes it is necessary to stimulate the increase in dimension by injecting a defined quantity of $\mathrm{Ne}$ impurities, that stiffen the profiles and stimulate the island size increase.
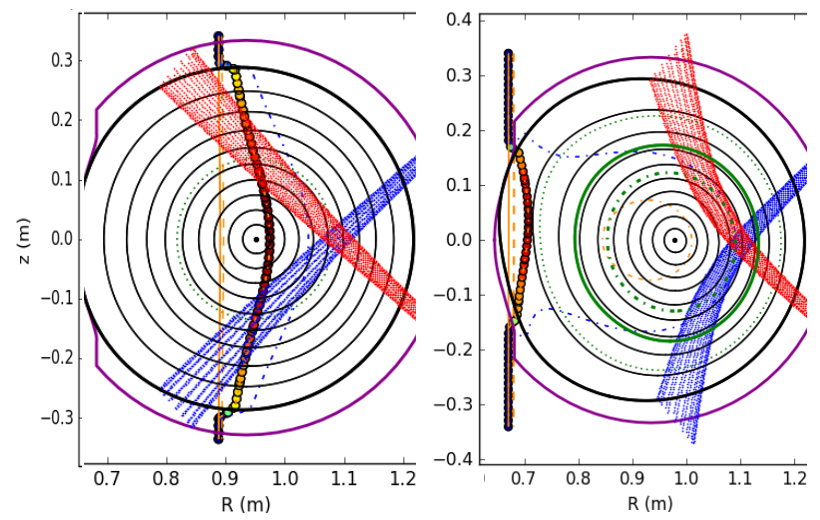

Fig. 1. Left: Plasma equilibrium and main EC resonances when operating at 4.7 field in the vessel centre. Probe beam (in red) is launched from the bottom right side, while the beam is received at the top right side (in blue) intersecting the probe on the equatorial plane. Right: the same with magnetic field at 3.6 $\mathrm{T}$ in the vessel center. The thin vertical (solid and dashed) lines correspond to the EC cold and warm resonances, while the Upper Hybrid (UH) resonance is marked by dots in colour.

In fact, the theoretical predictions of possible presence of low-threshold PDI rely on the possible inversion of the local density gradient profile, as in a well-grown island. The scope of the experiments is thus to verify the existence of the phenomenon and possibly quantify the power or plasma density threshold for its occurrence.

\subsection{Probe launch and receiving conditions}

The $140 \mathrm{GHz}, 350 \mathrm{~kW}$ gyrotron beam used as the probe (in red in Fig.1) is launched from the lower mirror of the EC launcher located in FTU port 8, while the radiation is collected from the upper mirror of the same launcher. The launcher is set to an angle such that the received beam (in blue in Fig.1) intersects the probe on the equatorial plane (symmetric configuration) or, for some shots at $4.7 \mathrm{~T}$, where the probe beam (launched horizontally) intersects the EC resonance (asymmetric configuration). The probe is launched with a toroidal angle of $5^{\circ}$ in co-current direction, and usually the probe is swept toroidally back and forth from $1^{\circ}$ to $9^{\circ}$ in 200 $\mathrm{ms}$, to put in evidence variations in the received signal with different amounts of beam overlapping, that could be taken as indication of emissions located in the superposition or "scattering" volume.

\subsection{Receiver setup}

In a first stage of experiments only one radiometer frontend was available, and it was set to receive the $\mathrm{O}$ polarized wave; later a second front-end was connected to the line adding a wire-grid to split the polarization, and it was usually set to receive the $\mathrm{X}$ polarized wave. The radiation sent to the first radiometer was modulated at around $10 \mathrm{kHz}$ to allow (constant) background noise subtraction in the subsequent conditioning by the filter bank section. The second radiometer was not modulated, in order to have a clean IF signal, to allow analysis of the Fast Digitizer signals free from modulation effects.

\subsection{Data treatment and calibration}

The calibration is done acquiring spectra from Ohmic shots (without EC power injection) in which the receiver line of sight intersects the EC resonance in an optically thick plasma region of known electron temperature, and, in the case of the digitizer raw data, also subtracting an offset due to noise, measured by receiver when blinded; details are given in [9]. A comprehensive database of observation has been realized, including all the performed shots, their main plasma parameters and for some of them, as temperature and density, the global (averaged) and local values (in the plasma centre and in the scattering volume). Also the main MHD island present in the plasma, m:n $=2: 1,3: 2$ or $3: 1$ modes has been recorded, together with the amplitude of the reference Mirnov Coil signal. This allowed the analysis of the statistical occurrence of the observations with respect to one or two chosen parameters.

\section{Emissions detected by CTS}

Several emissions of different type were detected in nearly 200 shots performed from 2014 to 2017 . Some of them were found to be gyrotron side emission lines, detected also in the stray radiation picked-up in dedicated shots [10]. Other ones were found in $7.2 \mathrm{~T}$ shots performed in 2014, and reported in [8]; they may be of the same origin as those reported here, even though this has still to be confirmed by new experiments to be performed at 7.2 T. Here below we describe the emissions found in the latest experimental campaigns, and the studies of correlation of these emissions with the MHD activity (in particular, with the islands rotation frequency) and with the evolution of the other plasma parameters. In order to facilitate the description, the emissions have been divided in a few types, numbered as in Table 1, where a synthetic description is given. In the next sections we will describe a limited set of emissions, which have characteristics closer to the ones expected by PDIs and with enough statistical occurrence. In this respect, we concentrate on line emissions of types 2 , and 7 , since the description of emission of type 3 have not enough observations, and emissions of type 1, 4 and 6 correspond to the emissions already described in [8], respectively in sections $4.2,4.1$ and 4.3 . 
Table 1. Types of observed emission. Emissions of Type 2 and Type 7 are the ones studied in more detail in this paper.

\begin{tabular}{|c|c|c|c|c|}
\hline n. & $\begin{array}{c}\text { Type of } \\
\text { spectrum }\end{array}$ & $\begin{array}{c}\text { Min. } \\
\text { duration } \\
(\mu \mathrm{s})\end{array}$ & $\begin{array}{c}\text { Max } \\
\text { persistence } \\
(\mathrm{ms})\end{array}$ & $\begin{array}{c}\Delta \text { frequency } \\
\text { or range } \\
(\mathrm{GHz})\end{array}$ \\
\hline 1 & $\begin{array}{c}\text { bursts of } \\
\text { continuum }\end{array}$ & $<1$ & variable & $>( \pm) 1$ \\
\hline 2 & line & $<20$ & $10-20$ & $(-)(0.2-0.4)$ \\
\hline 3 & broad line & $>50 ?$ & $\sim 5$ & $\pm(0.2-0.3)$ \\
\hline 4 & $\begin{array}{c}\text { line of } \\
\text { variable } \\
\text { frequency }\end{array}$ & $>1$ & $>20$ & $-(0.5-1.2)$ \\
\hline 5 & continuum & $<50$ & $<10$ & $-(0.05-0.2)$ \\
\hline 6 & $\begin{array}{c}\text { multiple } \\
\text { lines }\end{array}$ & $<50$ & $>15$ & $\pm(0.05-0.4)$ \\
\hline 7 & line & $<100$ & $<100$ & $( \pm)(0.6-0.7)$ \\
\hline
\end{tabular}

\subsection{Emissions from the gyrotron}

As already reported in [10], in the spectra there are often side emissions that are originated by the gyrotron. These are put in evidence in the spectra taken when the receiving line was disconnected from the antenna and picked up the gyrotron stray radiation outside the launcher. This was presently done offline, with dedicated shots. A line with variable frequency, one $\mathrm{GHz}$ above the gyrotron frequency, is often found. Other emissions of the same appearance have been presently excluded from the analysis, waiting to confirm if they are originated (or not) from the gyrotron. With a deeper observation of the spectra of the stray radiation, in fact, also the emissions reported in section 4.4 in [8] as "periodic bursts at around $15 \mathrm{MHz}$ from the probe" were observed in the gyrotron spectrum, thus revealing the weakness of the assumption of having a clean gyrotron spectrum, unless it is measured with the same highsensitivity diagnostic apparatus.

With the future installation of a new receiving line, the aim is to receive simultaneously in two different channels the emission from the plasma and the emission from the gyrotron, to exclude any possible misinterpretation of the origin of the emissions, that may also be due to gyrotron spectrum disturbances, induced by reflections or scattering of power into the plasma, that cannot be excluded in the present analysis.

\subsection{Emissions of Type 2}

These emissions (Fig.2) appear in shots with $m: n=2: 1$ MHD rotating mode in different field configurations. The frequency indicated in the spectrogram is the frequency distance (in absolute value) from the local oscillator (LO) frequency, usually tuned on the gyrotron one. In this case we cannot distinguish the emissions higher or lower than the gyrotron frequency, since they appear superimposed. Occasionally the LO frequency is significantly shifted from the gyrotron frequency (as in the shot in Fig.12) to distinguish the upper and the lower sidebands from the probe.

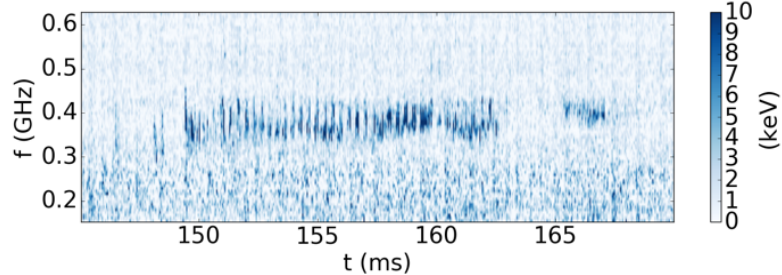

Fig. 2. Example of line-emission of Type 2 (shot \#40753).

Type-2 emissions are more frequent (see Fig.3) at higher densities and when a higher $\mathrm{dB} / \mathrm{dt}$ of the rotating island is measured by the reference Mirnov coil, i.e. when the magnetic island is larger, and thus the density gradient can be more easily found inverted, condition to facilitate the PDI occurrence as predicted by theory $[6,7]$.

Electron density in scattering vol. $\left(10^{20} \mathrm{~m}^{-3}\right)$ vs MHD amplitude

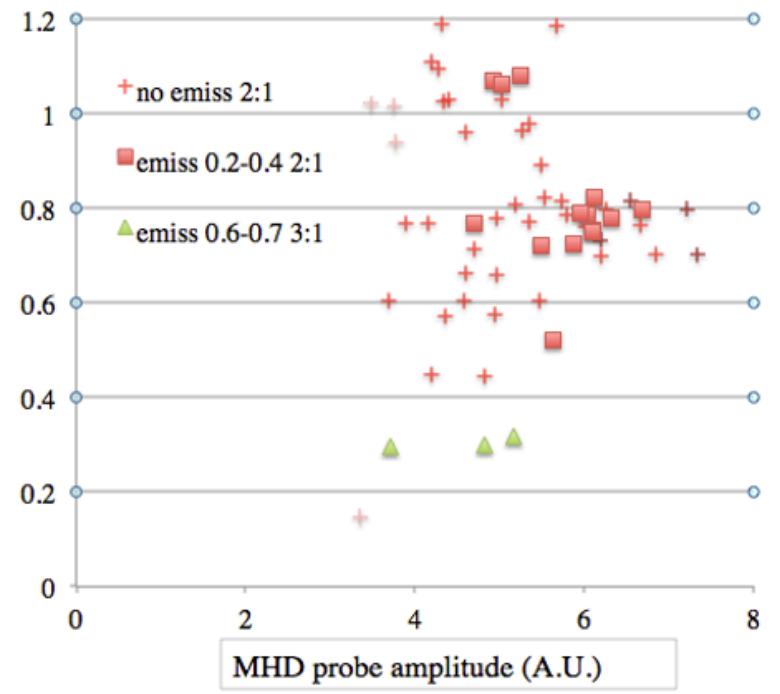

Fig. 3. Distribution in density and MHD amplitude of shots with emission of Type 2 (squares) and Type 7 (triangles) with respect to those without emission (crosses).

In any case emissions appear as spiky, with a few microseconds time-scale of temporal evolution (Fig.4).

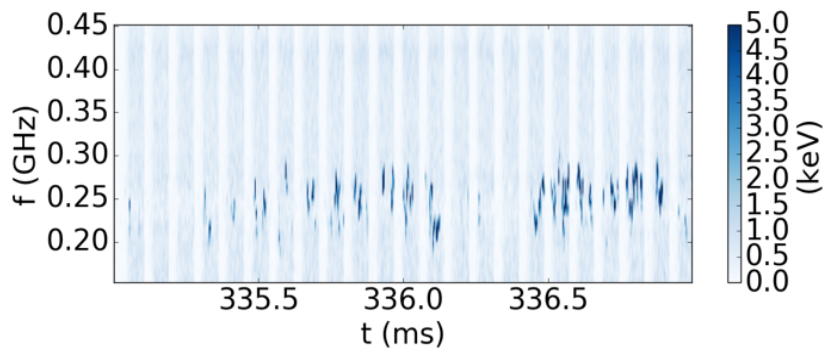

Fig. 4. Fast time-behavior of Type 2 emissions (shot \#40772).

Emissions seem linked with the presence of a $m: n=2: 1$ MHD island and their characteristic frequency shift may vary during the plasma evolution (from $0.25 \mathrm{GHz}$ to 0.3 $\mathrm{GHz}$ and back to $0.2 \mathrm{GHz}$, as in the case of shot \#40772, in Fig.5, Top). In this particular case, the change may be correlated to the probe in the poloidal direction by a 3 degrees angle in $30 \mathrm{~ms}$. 


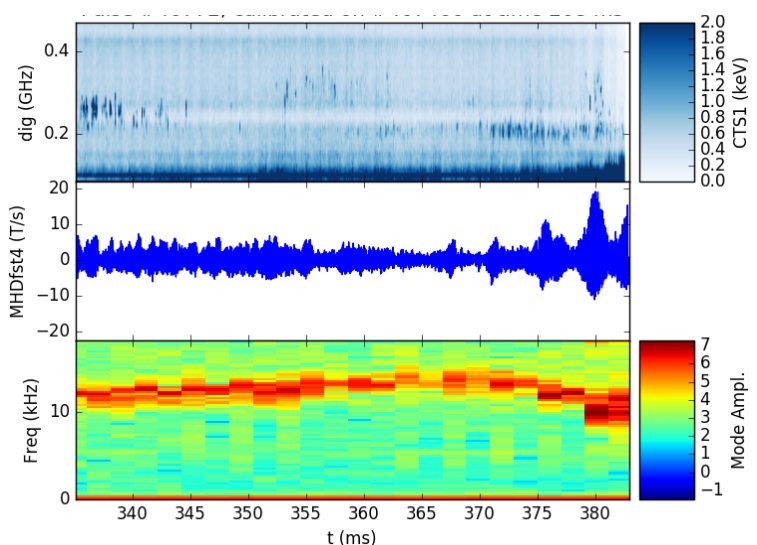

Fig. 5. Top: emissions for shot $\# 40772$, showing a variable frequency. Center: Mirnov coil signal, whose frequency spectrum (Bottom) indicates the presence of a $m: n=2: 1$ mode rotating at a frequency above $10 \mathrm{kHz}$.

We studied the correlation of the emission amplitude with the Mirnov coil signal, to understand if the time evolution can be due to the rotation of the island. For this aim, their statistical appearance (average of power in the frequency band) was evaluated as a function of time and compared with the rotating MHD island phase (Fig.6). In two cases (\#40771 and \#40772) of asymmetric launch no correlation was found with the rotation frequency, while in another case, for a shot with symmetric launch at a relatively low density for FTU $\left(0.3 * 10^{20} \mathrm{~m}^{-3}\right.$, \#40753) during plasma current ramp-up, the variation of the statistical appearance of the emissions seems to be correlated with the island rotation (Fig.6).

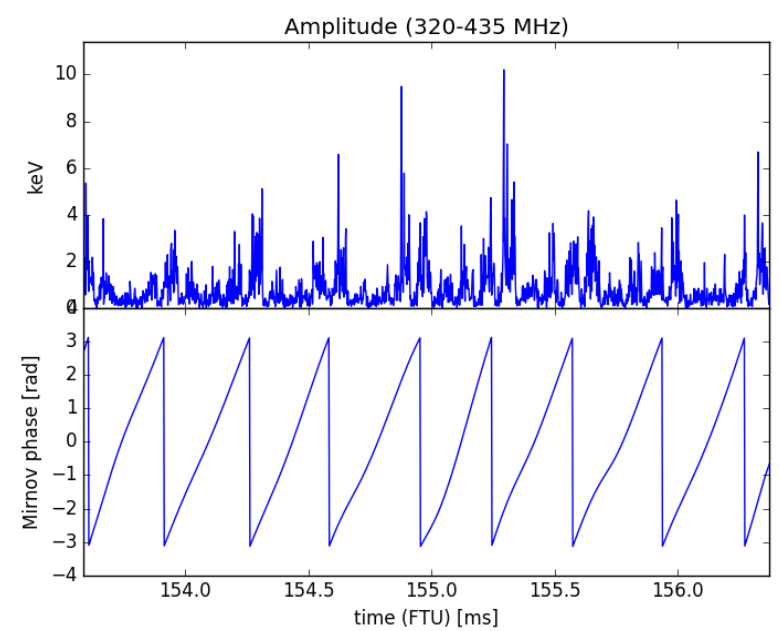

Fig. 6. Amplitude of emission between $0.32-0.435 \mathrm{MHz}$ from the probe (Top), compared to the phase of the Mirnov coil signal (Bottom) for shot \#40753: for this shot a correlation is found, while in other ones (\#40771 and \#40772) it is not.

Moreover, for shot \#40753 the time spectrum of the amplitude of the line emission shows a trend similar to the MHD rotation evolution, i.e. slows down during the island rotation slowing down (Fig.7). In other terms it seems that the emissions are more frequent (or stronger) every time the island is in the same particular position during its rotation, even when changing rotation frequency.

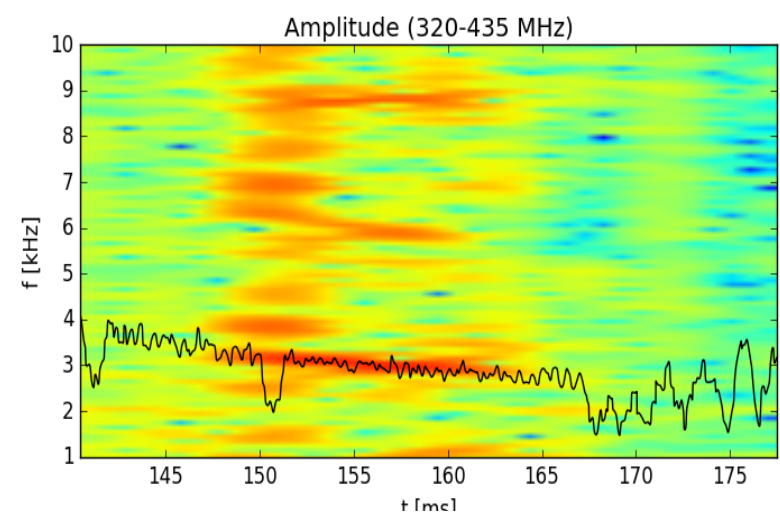

Fig. 7. Frequency spectrum of the emission amplitude in time, with the frequency of the Mirnov coil (black line) superimposed, showing a correlation of the variation of the averaged intensity of the emission with the rotation frequency of the island, during the island slowing down, for shot \#40753

The configurations of the shots where a correlation is observed is different from the one in which it is not found. The launch configuration for shots \#40771 and \#40772, was asymmetric: the probe beam intersects the $m: n=2: 1$ magnetic island in 2 points: the first in the low field side, close to the $\mathrm{UH}$ resonance position and then again at the EC resonance (Fig.8).

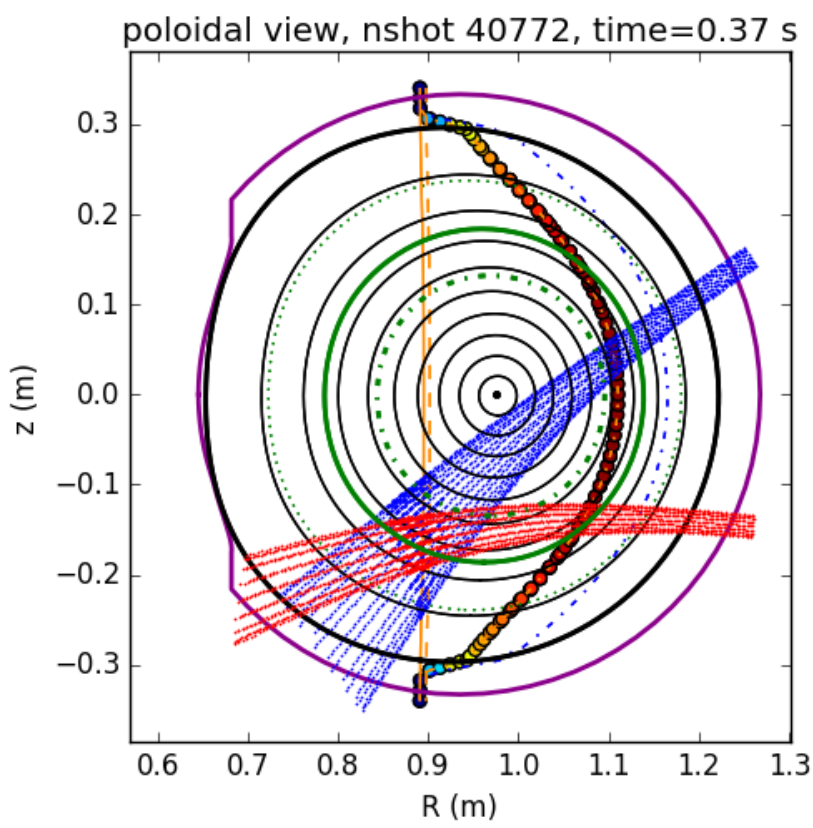

Fig. 8. Plasma equilibrium and resonances for shot $\# 40772$ with asymmetric launch: the probe beam intersects the island close to the $\mathrm{UH}$ resonance and then again the $\mathrm{q}=2$ surface (solid circle in green) close to the EC resonance. The thin vertical (solid and dashed) lines correspond to the EC cold and warm resonances, while the UH resonance is marked by dots in colour. The $\mathrm{q}=1.5$ and $\mathrm{q}=3$ magnetic surfaces are also shown as dashed-dotted lines.

Moreover for these shots the temperature profile is rather flat. In the case of shot \#40753 instead, the launching and plasma configuration is symmetric (Fig.9). The receiving line of sight crosses the launched beam close to the position of the $\mathrm{q}=2$ magnetic surface. The position of the $\mathrm{UH}$ resonance is also close to the crossing. 


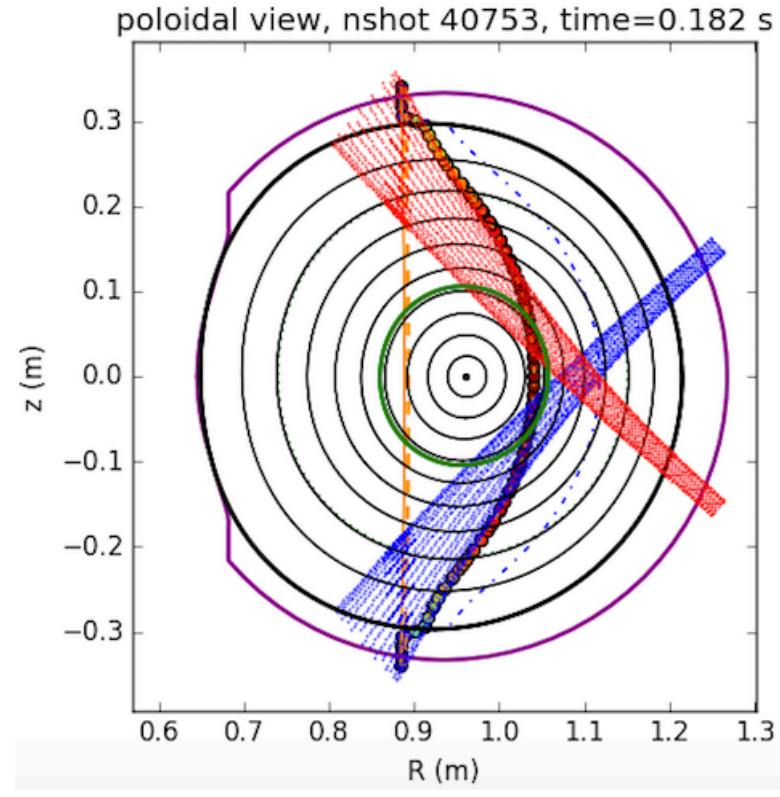

Fig. 9. Plasma equilibrium and main EC resonances for shot \#40753 with symmetric launch: the probe beam (in red) and received beam intersect themselves close to the estimated position of the $\mathrm{q}=2$ resonant surface on the equatorial plane, and not far from the UH resonance.

This is not the only difference from the shots \#40771 and $\# 40772$ : Also the temperature profile in the case of shot \#40753 is much more peaked (see Fig.10). This suggests a possibly different mechanism or a different location of the Type 2 emissions in the two cases. The superposition of resonances and magnetic rational surfaces (the $q=1.5$ and 3 are also shown in Fig. 8 and Fig.9 as dashed-dotted lines) increases the complication of the analysis aimed to find an explanation for the observed phenomena.

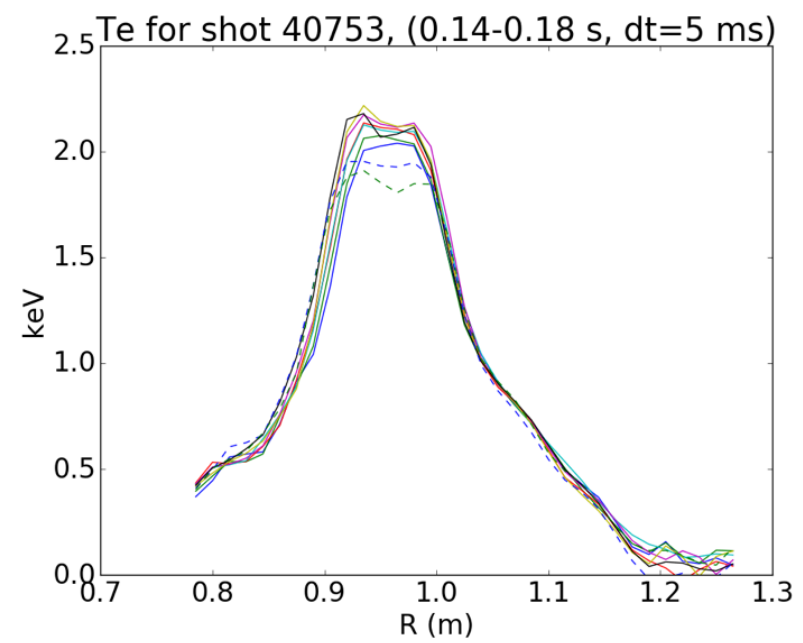

Fig. 10. Electron temperature profiles at different times during the Type 2 emissions of shot $\# 40753$, as reconstructed by the Michelson Interferometer.

In all cases of Type 2 emissions at $4.7 \mathrm{~T}$, the frequency shift with respect to the probe is lower than the Lower Hybrid (LH) frequency in the plasma core. Emissions of Type 2 are found also in $3.6 \mathrm{~T}$ shots, with a different launch configuration and at higher densities. Shot
$\# 40282$ is an example: for this shot (as for \#40771 and \#40772) no strict correlation of the emissions with the island rotation has been found, despite the presence of a $\mathrm{m}: \mathrm{n}=2: 1$ MHD mode located at the beam crossing (see Fig.11). The UH resonance is not crossed by the beams but after a first bounce at the vessel. Also the LH cutoff is close to the crossing and the island position, and this may influence the emissions or the clean reception of the signal, since the (small and unwanted) part of the beam injected in the $\mathrm{X}$ polarization may be reflected in the direction of the received beam, even if a toroidal angle is given to the probe to prevent direct reflection.

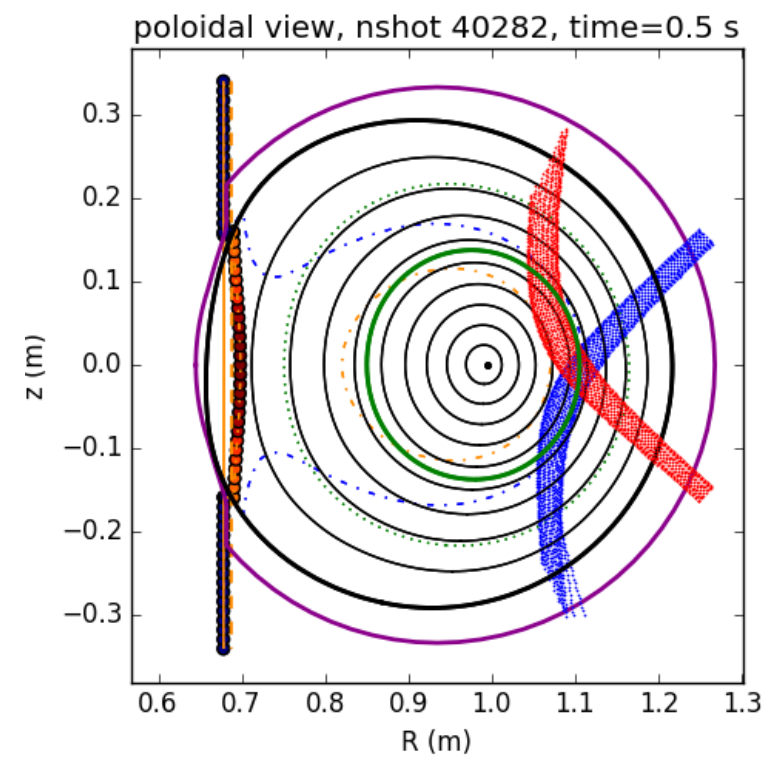

Fig. 11. Plasma equilibrium and main $\mathrm{EC}$ resonances for shot \#40282 with symmetric launch: the probe beam (in red) and received beam intersect themselves close to the $\mathrm{q}=2$ resonant surface on the equatorial plane and are refracted toward the vessel by the high-density plasma.

In this case, due to the shifted LO frequency, the emission is clearly seen only below the probe frequency (line at $0.8 \mathrm{GHz}$ ) at around $0.5 \mathrm{GHz}$ from the $\mathrm{LO}$ frequency, as seen in Fig. 12.

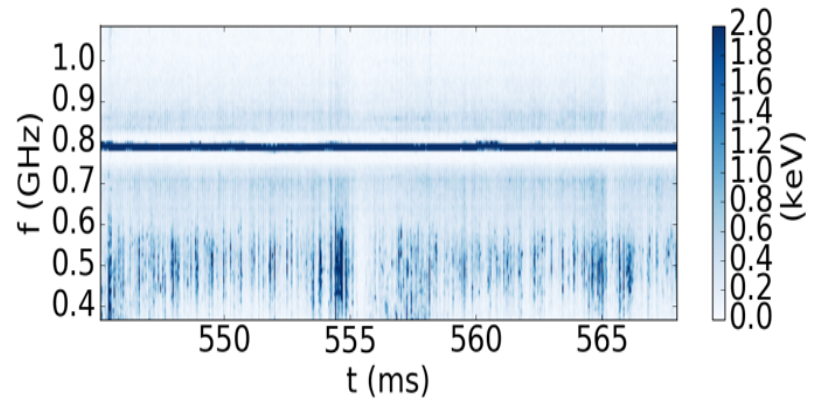

Fig. 12. Emissions below the probe frequency (shot \#40282).

\subsection{Emissions of Type 7}

Another line emission type that is candidate as due to PDIs is Type 7. It has an intermittent character (as seen in Fig.13, where a $10 \mathrm{kHz}$ chopping on the incoming radiation is also modulating the entire spectrum). 


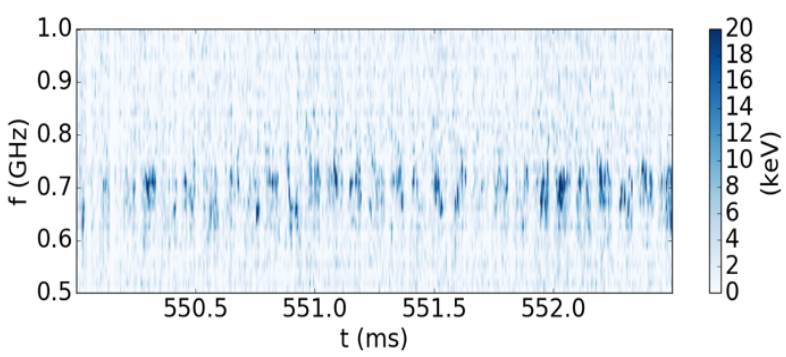

Fig. 13. Example of line-emission of Type 7 (shot \#40735).

This emission is found in a few shots at lower density (triangles in Fig.3), characterised by the presence of a $\mathrm{m}: \mathrm{n}=3: 1 \mathrm{MHD}$ island and by hollow temperature profiles (Fig.14).

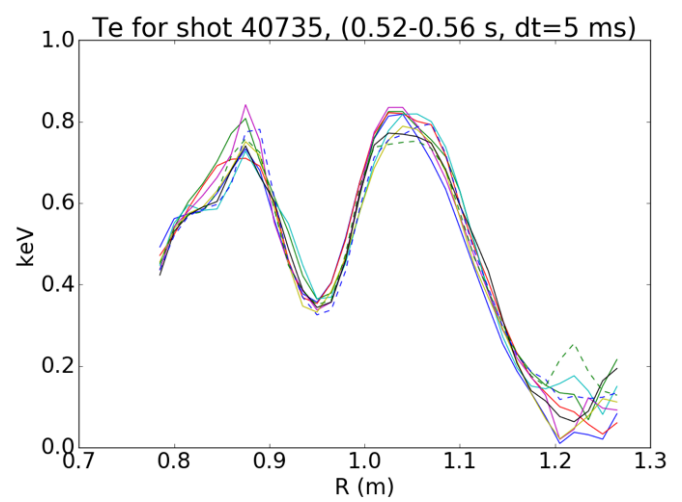

Fig. 14. Electron temperature profiles at different times during the Type 7 emissions of shot $\# 40735$, as reconstructed by the Michelson Interferometer. The regions at major radius $\mathrm{R}=0.83$ $\mathrm{m}$ and $\mathrm{R}=1.08 \mathrm{~m}$ are perturbed (flattened) by the presence of a $\mathrm{m}: \mathrm{n}=3: 1$ MHD magnetic island.

The presence of a $m: n=3: 1$ MHD rotating island is confirmed by the analysis of the phases of Mirnov coils signals collected in different toroidal positions. The reconstructed launch and plasma equilibrium configurations are shown in Fig.15. In this case the receiving beam crosses the probe beam close to the $q=3$ rational surface where the MHD island is located. Before reaching the EC resonance (where they are presumably damped) the beams intersect again the $\mathrm{q}=3$ surface, in the proximity of the UH resonance. For these shots the frequency shift of the detected emission is compatible with the LH frequency in the plasma core. The reconstruction of the density profiles for these shot is more complex, and will require a dedicated work.

\section{Conclusions}

The interpretation of the observations in terms of effects of secondary waves generated by Parametric Decay of the probe is still uncertain, given also the many way that the radiation can reach the resonances, for example after bouncing on the tokamak vessel. Certainly a comprehensive set of evidences point to phenomena occurring during probe injection (and thus during EC beam launch for heating and Current Drive) that can be studied with a diagnostic able to detect side emissions at a level of a the plasma thermal emission with a good spatial and a very high temporal/frequency resolution, as the improved CTS diagnostics recently operating on different devices. To locate and discriminate them from the gyrotron side emissions, independent "reference" receiving lines from plasma lines of sight and from the gyrotron stray radiation are necessary. A new receiving line, with an independent access on the equatorial plane and (alternatively) the capability to collect the gyrotron stray radiation before injection into the plasma [11] is presently installed on the FTU and connected to the CTS diagnostics, for future deeper investigations.

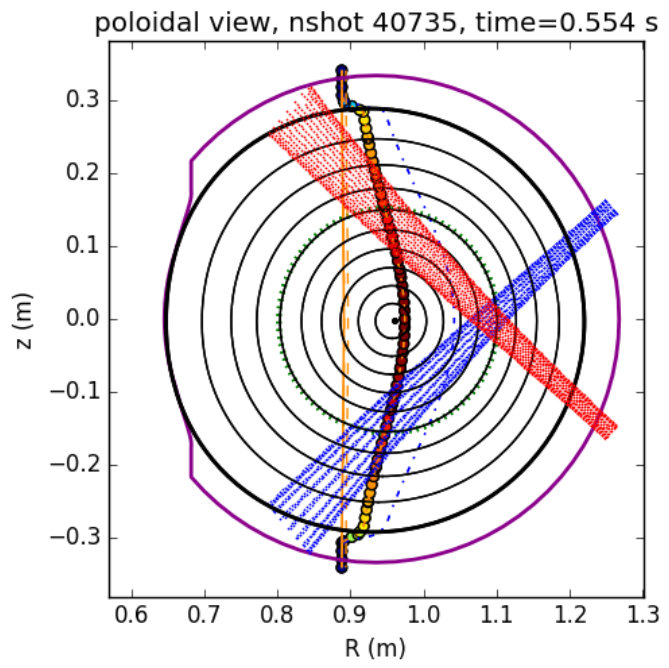

Fig. 15. Plasma reconstructed equilibrium and resonances for shot \#40735 with symmetric launch: receiving beam crosses the probe beam close to the $\mathrm{q}=3$ rational surface (dotted circle in green) where a MHD island exists. Before reaching the EC resonance (thin vertical line) the beams intersect again the $q=3$ surface, in the proximity of the UH resonance (dots in colour).

This work has been carried out within the framework of the EUROfusion Consortium and has received funding from the Euratom research and training programme 2014-2018 under grant agreement No 633053. The views and opinions expressed herein do not necessarily reflect those of the European Commission.

\section{References}

1. W. Bin et al., JINST 10, P10007 (2015)

2. W. Bin et al., Rev. Sci. Instr. 87, 11 E507 (2016)

3. W. Bin et al., Fus. Eng. Des. 96-97, 733-737 (2015)

4. E. Z. Gusakov and A Y Popov, Plasma Phys. Control. Fusion 60, 025001 (2018)

5. S. K. Hansen, et al., Plasma Phys. Control. Fusion 59, 105006 (2017)

6. E. Z. Gusakov, et al., Plasma Phys. Control. Fusion 59, 075002 (2017)

7. E. Z. Gusakov and A. Y. Popov, Phys. Plasmas 25, 012101 (2018)

8. A. Bruschi, et al., Nucl. Fusion 57, 076004 (2017)

9. B. Baiocchi et al, ECPD 2017, (submitted to JINST).

10. A. Bruschi, et al., EPJ Web of Conferences 149, 03017 (2017)

11. O. D'Arcangelo et al, JINST 13, C01012 (2018) 